\title{
Pour une planification méthodique des activités de formation
}

\author{
Pierre JEAN
}

\begin{abstract}
Résumé Contexte: Les formations font rarement l'objet d'une planification méthodique, les contenus disciplinaires prenant généralement plus d'importance que l'approche pédagogique.

But : Proposer une démarche systématique en sept étapes pour la planification méthodique des activités de formation. Illustrer la démarche à partir de l'exemple d'une formation générale en pédagogie médicale.

Méthode : La démarche a été développée à partir des modèles classiques de la planification des activités de formation et a été mise au point au cours de plusieurs années d'expérience dans la formation de formateurs en santé des pays du Nord et du Sud. Aujourd'hui, cette démarche se déroule de la façon suivante : 1- Définition des tâches professionnelles; 2- Détermination des compétences à acquérir pour accomplir les tâches professionnelles; 3-Analyse des besoins de formation ; 4- Formulation les objectifs d'apprentissage ; 5- Choix des moyens d'apprentissage ; 6-Evvaluation des apprentissages; 7-Évaluation de l'activité de formation.

Conclusion : Que l'on ait à planifier un atelier de formation continue d'une journée ou l'ensemble d'un programme de formation générale en pédagogie médicale, on a intérêt à adopter une approche méthodique.
\end{abstract}

Mots clés Planification méthodique des activités de formation.

Summary Context: Training in medicine is not always the result of systematic planning, more attention being generally paid to contents rather than to pedagogy.

Aim : A systematic approach to designing instruction is proposed using, as an example, a faculty development program in medical education.

Method: Procedures were first developed according to existing models of systematic educational planning. Then, over the years, they were adapted to the training of trainers in North American and developing countries. At present, they unfold into the following seven steps: 1-Listing of professional tasks to be accomplished by the trainee; 2-Identification of competencies required to accomplish those professional tasks; 3-Analysis of learning needs; 4-Formulation of learning objectives; 5-Selection of learning methods; 6-Assessment of learning; 7- Evaluation of the educational program.

Conclusion : Systematic educational planning is useful whether in designing a one-year training program in medical pedagogy or a one-day workshop in continuing medical education.

Key words Systematic instructional planning.

Pédagogie Médicale $2001 ; 2: 101-107$.

\section{Introduction}

Dans les facultés de médecine, on a longtemps cru que la seule maîtrise des contenus disciplinaires était suffisante pour faire de l'enseignant un bon éducateur. On s'inquiétait peu de savoir comment l'étudiant ou le médecin en exercice apprenait, de quelle façon on devait planifier ses apprentissages pour qu'ils soient efficaces, comment mettre de l'avant des stratégies éducatives performantes, comment mieux évaluer les connaissances, les compétences et les performances et enfin, comment évaluer une activité éducative. Bref, l'enseignant se contentait de transmettre son savoir en imitant le mieux possible ceux qui l'avait formé. C'est ainsi que plusieurs parmi nous sommes devenus enseignants du jour au lendemain, sans préparation particulière pour cette fonction.

Depuis plus de cinquante ans, l'enseignement médical

Unité de santé internationale - Université de Montréal - 2375 chemin de la Côte Ste Catherine bureau 7072 - C.P. 6128 , succursale Centre ville - Montréal, Québec, H3C3J7, Canada Tél. : (514) 739-4631 - Fax : (514) 739-4631 - Adresse électronique : pierre.jean@citenet.net 


\section{Références}

s'est ouvert aux sciences de l'éducation et de la psycho$\operatorname{logie}^{1}$. Peu à peu, on a accepté l'idée que les enseignants doivent non seulement maîtriser certaines techniques d'enseignement, mais encore être familiers avec les fondements de la pédagogie adaptée à la médecine. De plus, sans nécessairement être eux-mêmes des chercheurs en pédagogie médicale, ils doivent être capables d'interpréter et d'utiliser les résultats de la recherche dans l'exercice de leur profession.

Au cours des dernières années, la recherche en pédagogie médicale a fait des progrès considérables. Bien entendu, comme le souligne Bordage ${ }^{2}$, la recherche est essentielle à la planification et à l'évaluation de la formation en médecine. Toutefois, pour que les résultats de la recherche aient un impact sur la formation, certaines conditions sont nécessaires. Entre autres, il faut que les enseignants puissent faire le lien entre la théorie et la pratique, entre la recherche et le terrain. Ils assureront ce lien de façon plus efficace s'ils sont capables de planifier de façon méthodique chacune de leurs interventions éducatives. Cet article propose au lecteur une démarche méthodique en sept étapes pour la planification de formations en médecine. La démarche a été utilisée dans l'élaboration de plusieurs programmes de formation pédagogique de formateurs tant dans les pays du Nord ${ }^{3}$ que ceux du Sud $\mathrm{d}^{4}$. L'un de ces programmes ${ }^{5}$ servira d'ailleurs à illustrer chacune des étapes de la démarche proposée.

\section{Nécessité d'une planification méthodique des activités de formation}

Pour être efficace, un apprentissage doit avoir fait l'objet d'une planification rigoureuse. On prend bien la peine de planifier les interventions dans tous les autres domaines de la vie, qu'elle soit professionnelle ou personnelle. Que penserait-on d'une recherche qui n'aurait pas été planifiée au préalable, qui ne suivrait pas un protocole établi à l'avance? Que dire d'une équipe médicale qui travaillerait sans plan de traitement? De même, dans les programmes de santé publique, on ne saurait commencer l'implantation d'une intervention auprès d'une population sans qu'auparavant elle ait fait l'objet d'une planification appropriée.

Il existe plusieurs modèles ${ }^{6,7,8}$ pouvant servir à une planification méthodique des activités de formation. Au cours des années, s'inspirant de ces modèles et de l'ex-
Tableau : Étapes de la planification méthodique d'une formation générale en pédagogie médicale

1- Définir les tâches professionnelles que les enseignants auront à accomplir

2- Déterminer les compétences à acquérir pour accomplir les tâches professionnelles

3- Analyser les besoins de formation

4- Formuler les objectifs d'apprentissage

5- Choisir les moyens d'apprentissage

6- Évaluer les apprentissages

7- Évaluer l'activité de formation

périence acquise lors de la formation pédagogique des enseignants en santé, une démarche en sept étapes a été progressivement mise au point (Tableau). À l'usage, cette démarche a subi certaines modifications. Dans les années 1980, on accordait beaucoup d'importance aux objectifs d'apprentissage. Puis, dans les années 1990, c'est l'analyse des besoins de formation qui a reçu une attention particulière. C'est cette l'analyse des besoins qui figure au tout début de la planification systématique des activités de formation, dans l'ouvrage utilisé à cette époque et intitulé "Apprendre à enseigner les sciences de la santé " ${ }^{\text {. }}$

Enfin, plus récemment, on a réalisé que la démarche se déroulait de façon plus méthodique si on commençait l'exercice de planification par un relevé détaillé des tâches professionnelles concrètes qui devront être accomplies après la formation ${ }^{5}$.

\section{Première étape: Définir les tâches professionnelles que les enseignants auront à accomplir}

Si l'on veut qu'une formation soit vraiment utile, sa planification doit partir de réalités concrètes, à savoir, des tâches que l'apprenant accomplira durant l'exercice quotidien de sa profession. L'adulte apprend de façon plus efficace lorsqu'il sait au départ ce qu'il fera à court terme avec ce qu'il aura appris. Sa volonté d'apprendre s'oriente davantage vers les tâches à accomplir dans son 
milieu social ; il sent le besoin d'appliquer immédiatement ce qu'il vient d'apprendre ${ }^{10}$. Pour lui, il ne suffit pas d'apprendre simplement pour apprendre. Il a besoin de savoir quelle tâche précise il pourra mieux accomplir au terme de son apprentissage. Dans ces conditions, il est tout à fait pertinent de commencer la planification méthodique des activités de formation par une description détaillée des tâches professionnelles à accomplir.

Dans le cas de la formation pédagogique des formateurs choisie pour illustrer la planification méthodique des activités de formation, le relevé des tâches professionnelles a été effectué à l'aide de la technique du journal de bord.

On a demandé à un certain nombre de formateurs en exercice d'inscrire sur une feuille les actions qu'ils avaient menées à chaque heure d'une journée représentative de leur vie professionnelle. Auparavant, on avait pris soin de définir ce que l'on entend par une tâche, à savoir, un travail particulier et spécifique qui doit être fait.

Voici quelques-unes des tâches mentionnées dans le journal de bord de certains formateurs : préparation d'un atelier de formation à l'intention d'agents sanitaires du district ; animation d'une séance de sensibilisation de parents de la communauté à la nécessité de la vaccination; rencontre avec le directeur du Service de la population du ministère pour expliquer une nouvelle approche en formation continue des personnels de santé; rédaction d'un rapport d'évaluation d'un programme de formation; rencontre avec un groupe d'agents sanitaires pour connaître leurs attentes face à un séminaire en santé reproductive; rédaction des cas à étudier lors d'un atelier à venir ; préparation de questions d'examen ; lecture d'un article consacré aux principes de l'éducation des adultes, etc.

Après avoir recensé un grand nombre de tâches concrètes du formateur, on peut regrouper celles-ci sous différentes rubriques. Ainsi, la préparation d'un atelier, d'une leçon ou d'un stage relève du même groupe de tâches, à savoir la planification d'une activité de formation.

C'est la préparation d'un atelier qui sera retenue pour illustrer les étapes subséquentes de la planification méthodique des activités de formation.

\section{Deuxième étape : Déterminer les compétences à acquérir pour accomplir les tâches professionnelles}

À partir des tâches regroupées dans un certain nombre d'activités, on a pu identifier les compétences que le formateur devra maîtriser pour les accomplir. Cette compétence, on l'a définie comme étant la capacité "d'utiliser ses connaissances, ses habiletés, ses attitudes et son jugement pour résoudre efficacement les problèmes qui se présentent dans l'exercice quotidien de la profession ${ }^{11}$. Il ne s'agit pas simplement d'attributs abstraits, mais de leur application dans des situations données. Dès maintenant, on s'aperçoit qu'il faudra tenir compte de ces situations au moment de choisir les moyens d'apprentissage. Il importe que le contexte de l'apprentissage se rappoche le plus possible de celui dans lequel les résultats de l'apprentissage seront utilisés.

Dans la planification de la formation choisie pour illustrer l'approche méthodique, il a été relativement facile de déterminer les compétences nécessaires à l'accomplissement des tâches retenues et regroupées sous la rubrique "planification d'une activité éducative ". Par rapport à cette activité, le formateur sera compétent dans la mesure où il pourra utiliser ses connaissances, ses habiletés, ses attitudes et son jugement pour résoudre efficacement les problèmes qui peuvent se présenter à lui dans la préparation d'une telle activité éducative. Pour la préparer efficacement, il ne suffit pas de connaître les principes de l'éducation des adultes et de la planification méthodique des activités de formation, mais encore faut-il pouvoir appliquer ces connaissances à la préparation concrète d'un atelier dans un contexte donné.

\section{Troisième étape : Analyser les besoins de formation}

À cette étape-ci, il convient de s'assurer dans quelle mesure l'apprenant possède déjà les compétences requises pour accomplir les tâches professionnelles déterminées au départ. Il s'agit d'évaluer l'écart qui existe entre ce qu'il peut déjà faire et ce qu'il serait désirable qu'il puisse faire. Cet écart représente le 


\section{Références}

besoin de formation qu'une activité éducative devrait normalement combler. Un besoin se définit comme l'écart qui existe entre la situation actuelle et la situation désirée. De manière générale, il s'exprime en termes de lacune ou de manque.

On utilise plusieurs techniques pour déterminer les besoins de formation, suivant la nature du besoin considéré. Ainsi, pour l'analyse des besoins démontrés ou observés chez les personnes à former dans le cadre de la formation générale en pédagogie médicale dont la planification sert à illustrer l'approche méthodique, la technique de l'Incident critique ${ }^{12}$ a été particulièrement utile. Un certain nombre d'enseignants ont été réunis à qui il était demandé de faire la liste d'incidents malheureux dont ils avaient été témoins et qui avaient abouti à des résultats non désirés lors de diverses formations. On est arrivé ainsi à relever des lacunes révélant les besoins de formation des enseignants. De façon générale, l'une des lacunes le plus souvent observée chez la grande majorité des enseignants est précisément reliée à la mauvaise organisation pédagogique des activités de formation.

Pour déterminer les besoins ressentis par les futurs participants à la formation générale en pédagogie médicale, ceux-ci ont été soumis à la technique du Groupe nominal ${ }^{13}$. À tour de rôle et par tours de table successifs, les participants ont énoncé, sans en discuter, ce qu'ils percevaient comme leurs lacunes les plus importantes dans l'exercice de leur fonction d'enseignant. De manière générale, une fois que le relevé des tâches à accomplir et des compétences à acquérir a été complété, les apprenants n'ont pas de difficulté à énoncer ce qui leur manque à cet égard. Cet exercice est particulièrement utile pour motiver les participants. C'est lorsque l'on devient conscient de ses lacunes que nâ̂ssent le désir et l'énergie d'apprendre.

Pour analyser les besoins normatifs ou institutionnels en regard de la formation pédagogique utilisée comme exemple, il a été possible de rencontrer les organismes gouvernementaux et non gouvernementaux des pays d'où allaient provenir les participants, et ceci, dès le début de la planification de la formation. À cette occasion, les partenaires institutionnels ont pu faire connaitre leurs attentes vis-à-vis du programme en voie de préparation.

\section{Quatrième étape: Formuler les objectifs d'apprentissage}

Pendant des années, on a attaché la plus grande importance aux objectifs d'apprentissage. À la suite de Mager on répétait "Si on ne sait pas où l'on va, on risque de se retrouver là où on ne voulait pas ». Que de collègues ont été détournés à tout jamais de la pédagogie parce qu'on les a forcés à écrire des objectifs qui malheureusement ne servaient que très peu. Or, il ne suffit pas de savoir où l'on va. Encore faut-il savoir pourquoi on y va! Si on ne sait pas pourquoi on va quelque part, on risque d'être fort déçu à l'arrivée. L'expérience a montré que la rédaction des objectifs prend tout son sens si elle découle des besoins de formation démontrés et surtout ressentis par ceux-là même qui doivent acquérir les compétences nécessaires pour accomplir des tâches professionnelles concrètes. Dans ces conditions, la rédaction des objectifs d'apprentissage devient non seulement facile mais pertinente, les objectifs étant rattachés au contexte de la vie professionnelle de la personne en formation. Les comportements désirés sont alors facilement observables et les conditions de réalisation des objectifs deviennent explicites. Les objectifs d'apprentissage prennent leur origine dans les compétences professionnelles à acquérir.

Dans l'exemple choisi pour illustrer l'approche méthodique de la formation générale en pédagogie médicale, voici l'un des objectifs qui a été formulé : "À la suite de sa formation pédagogique, l'enseignant sera capable de planifier de façon systématique un atelier d'apprentissage d'une journée à l'intention de collègues médecins dans le cadre de leur formation continue".

À la lecture d'un objectif, on devrait pouvoir imaginer, sans difficulté, les moyens les plus appropriés pour l'atteindre et la façon d'en évaluer l'atteinte. Si on a de la difficulté à le faire, c'est que l'objectif n'a pas été formulé selon les règles de l'art.

\section{Cinquième étape : Choisir les moyens d'apprentissage}

Par moyen, on entend tout ce qui permet d'atteindre un objectif. Ce terme englobe les méthodes et les techniques. Une méthode pédagogique se définit comme l'ensemble des principes et théories sur lesquels se fondent des activités d'apprentissage, alors que les tech- 
niques d'enseignement sont constituées par l'ensemble des outils utilisés pour mener à bien les activités de formation. En éducation des adultes, il va de soi que la méthode dite active est la méthode de choix. Dans cette méthode, l'enseignant permet à l'apprenant de trouver les solutions aux problèmes par ses propres moyens. À distinguer de la méthode affirmative, encore largement utilisée, dans laquelle l'enseignant connaît la réponse et se contente de la communiquer à l'apprenant.

Dans l'exemple ci-dessus, on a choisi l'atelier comme moyen pouvant permettre à l'enseignant en formation pédagogique d'apprendre à " planifier de façon systématique un atelier d'apprentissage d'une journée à l'intention de collègues médecins dans le cadre de leur formation continue ». Un atelier pour apprendre à planifier un atelier ! "The medium is the message ».

Par atelier pédagogique, on entend "un type de rencontre qui permet à des personnes d'échanger entre elles et avec des experts dans un climat de coopération, dans le but d'acquérir de nouvelles connaissances, habiletés ou attitudes sur des sujets ou des problèmes d'intérêt commun. C'est un mode d'intervention pédagogique ponctuel, intensif, dont la durée varie de quelques heures à quelques jours. Il est centré sur l'apprenant qui y a un rôle actif. Il est particulièrement efficace dans le domaine cognitif. Il trouve sa place en formation continue où on s'adresse à des professionnels en exercice. Sa planification, son organisation et sa réalisation demandent une préparation rigoureuse. " ${ }^{9}$ De manière générale, l'atelier est construit autour d'activités utilisant le modèle "Intrant - Pratique - Feedback ». Comme intrant, dans notre exemple cité plus haut, on a placé les participants en situation concrète : "Vous devez préparer un atelier d'apprentissage d'une journée à l'intention de médecins désireux d'améliorer leur façon d'annoncer une mauvaise nouvelle à leurs patients. De façon individuelle, rédigez le plan de l'atelier avant d'en discuter avec les collègues de votre groupe afin de présenter en plénière le plan retenu ». Une fois la commande placée (intrant), les participants ont l'occasion de faire l'exercice avec plus ou moins de succès (pratique) avant que l'animateur (ou un participant) fournisse ses commentaires et une synthèse (feed-back).

L'atelier pédagogique réunit les conditions qui favorisent l'apprentissage de l'adulte. On sait que l'adulte apprend dans la mesure où il est actif; où on fait appel à ce qu'il connaît déjà et à ses expériences; où le contexte dans lequel il apprend ressemble au contexte qui sera le sien lorsqu'il utilisera ce qu'il a appris ; où il pourra se servir dans un avenir prochain ce qu'il vient d'apprendre; où il reçoit sans tarder un feed-back sur ses performances; où il a une vision claire de ce qui est attendu de lui ; où il peut échanger avec ses pairs; où il est soumis à un stimulus dès le début de l'activité d'apprentissage ; où l'enseignant se soucie, non pas de ce qu'il enseigne, mais de ce que l'apprenant apprend ${ }^{14}$. Si l'atelier est largement utilisé dans la formation des formateurs proposée en exemple $e^{5}$ il n'est pas le seul moyen d'apprentissage adopté pour la formation pédagogique des enseignants. Pendant des années, et encore aujourd'hui, on a utilisé le module d'auto-apprentissage $^{9}$ : les participants doivent faire individuellement les exercices présentés dans dix-sept modules couvrant autant de thèmes fondamentaux de la pédagogie, et ceci, à raison d'un module par quinze jours ou trois semaines. À la suite de chaque module, ils se réunissent pour discussions et échanges avec quatre ou cinq collègues et un animateur. Cette formule est souple, chaque groupe pouvant fixer son propre calendrier de rencontres qui peuvent s'étendre sur une année.

\section{Sixième étape : Evaluer les apprentissages}

Un apprentissage ne saurait être valable s'il n'est pas évalué. Il existe un lien étroit entre les objectifs et l'évaluation de leur atteinte. Certains ont même suggéré de planifier l'évaluation des apprentissages immédiatement après la formulation des objectifs ${ }^{7}$. Cette étape est souvent la plus négligée, tout particulièrement en formation continue. Pourtant, elle est peut-être la plus importante.

Il est étonnant de constater que souvent les enseignants qui pourtant devraient servir de modèles aux étudiants, acceptent difficilement de voir évaluer leur propre apprentissage à la suite d'une formation pédagogique. Pour contourner cette difficulté, on a pris l'habitude de laisser aux enseignants en formation le choix des modalités de l'évaluation. Voici le résultat généralement obtenu : auto-évaluation et évaluation par les collègues, de même que par l'animateur, de la participation et des productions de chacun. Dans la formation générale en pédagogie, donnée en exemple, 


\section{Références}

l'animateur note les exercices individuels d'application, les lectures dirigées de même que la planification et l'exécution du projet éducatif personnel. L'auto-évaluation prend la forme d'un prétest et d'un post-test (identique au prétest) où l'on présente au participant une quinzaine de problèmes pédagogiques à résoudre. L'évaluation occupe une place importante dans toute formation. Des trois caractéristiques d'un instrument de mesure, à savoir la validité, la fiabilité et la commodité, c'est sur la validité que l'on met l'accent. Pour être valide, donc pertinente, l'évaluation, tout comme les objectifs, doit s'inspirer des tâches professionnelles à accomplir. Une attention particulière est portée à la congruence nécessaire entre les niveaux de l'objectif, du moyen d'apprentissage et de l'instrument de mesure. Si l'on veut que l'apprenant soit capable de faire face efficacement aux situations rencontrées dans l'exercice de sa profession, on doit lui offrir, durant son apprentissage et au moment de l'évaluer, de telles situations à résoudre. Il ne suffit pas d'évaluer ce qu'il sait, mais surtout ce qu'il est capable de faire avec ce qu'il sait.

Ainsi, pour évaluer dans quelle mesure le participant, à la fin de l'atelier, est capable de "planifier de façon méthodique un atelier d'apprentissage d'une journée à l'intention de collègues médecins dans le cadre de leur formation continue ", il lui a été demandé de remettre à l'animateur le plan de son atelier.

\section{Septième étape : Évaluer l'activité de formation}

Évaluer une activité éducative, c'est porter un jugement de valeur sur cette activité. Un tel jugement n'est possible qu'après avoir choisi, recueilli et interprété les renseignements pertinents à l'évaluation de l'activité. Pour que les résultats d'une évaluation soient crédibles, il importe de conduire cette opération de façon systématique, en respectant certaines normes établies. Tout comme l'ensemble de la formation, l'évaluation de celle-ci doit se faire selon une approche systématique. Elle doit être rigoureusement planifiée avant même que ne commence la formation.

Dans la formation pédagogique des enseignants on leur propose un modèle dérivé de celui de Stufflebeam ${ }^{15}$ dans lequel les questions d'évaluation sont groupées, à l'intérieur d'une matrice, sous quatre rubriques : le contexte, les intrants, le processus et les extrants. L'évaluation des apprentissages qui n'est qu'une des dimensions de l'évaluation de l'activité de formation, se place sous la rubrique des extrants.

Pour évaluer, non plus une formation générale en pédagogie médicale, mais une activité de courte durée, comme un atelier d'une journée, il est possible de procéder plus rapidement tout en s'inspirant du modèle mentionné ci-dessus. On demande aux participants d'écrire les aspects de l'atelier sur lesquels ils aimeraient faire des commentaires et des suggestions. Puis, à l'occasion de tours de table successifs, chacun énonce les points qu'il a choisis sans, à ce moment-ci, faire de commentaires, ni de suggestions. L'animateur enregistre au tableau les points en les classant sous les quatre rubriques du modèle "Contexte, intrants, processus et extrants ". Dans un deuxième temps, les participants sont appelés à formuler leurs commentaires et leurs suggestions en regard des points qu'ils ont retenus.

\section{Conclusion}

Pour qu'une formation soit pertinente, il importe que sa planification prenne en compte dès le départ les tâches concrètes qui devront être accomplies par les apprenants après leur formation. La description des tâches professionnelles débouche naturellement sur les compétences à acquérir pour les accomplir et, par la même occasion, sur les besoins de formation. Tâches, compétences et besoins de formation aboutissent à la formulation d'objectifs d'apprentissage exprimés en termes de comportements observables. Après quoi, les moyens d'apprentissage choisis, de même que les instruments d'évaluation, doivent fournir à l'apprenant un contexte qui ressemble au contexte de l'exercice professionnel. Finalement, l'évaluation de la formation doit faire, elle aussi, l'objet d'une planification rigoureuse, tout comme la formation elle-même.

Que l'on ait à planifier un atelier de formation continue d'une journée ou l'ensemble d'un programme de formation générale en pédagogie médicale, on a intérêt à adopter une approche méthodique. Dans les deux cas, la détermination des tâches professionnelles à accomplir facilite la planification des étapes subséquentes de la formation, étapes qui sont étroitement liées entre elles. 


\section{Références}

1. Miller G. Teaching and Learning in Medical Schools. Cambridge : Harvard University Press, 1961.

2. Bordage G. La recherche en pédagogie médicale en Amérique du Nord: tour d'horizon et perspectives. Pédagogie Médicale. $2000 ; 1$ : 9-12.

3. DesMarchais JE. Jean P. Delorme P. Basic Training Program in Medical Pedagogy : a 1 year program for medical faculty. Canadian Medical Association Journal. $1990 ; 142: 734-740$.

4. Jean P. Partenariat pour la formation de formateurs en santé reproductive : réflexions d'un éducateur depuis l'Afrique subsaharienne. Présentation à la Conférence internationale francophone en sciences de la santé, Moncton, 26 - 29 juin 1999.

5. Jean P. Formation pédagogique pour les formateurs en sciences de la santé et en santé reproductive. Montréal: Éditions de l'Unité de santé internationale de l'Université de Montréal, 2000.

6. Tyler RW. Basic Principles of Curriculum and Instruction. Chicago : The University of Chicago Press, 1949.

7. Guilbert JJ. Guide pédagogique pour les personnels de santé. Genève: Publication de l'Organisation Mondiale de la Santé, 1990.

8. McGaghie WC. Miller GE. Sajid AW. Telder TV. Introduction à un enseignement médical fondé sur l'acquisition de compétences. Genève: Publication de l'Organisation Mondiale de la Santé, 1978.
9. Jean P. DesMarchais JE. Delorme P. Apprendre à enseigner les sciences de la santé : Guide de formation pratique. Montréal: Les Publications Médéva, 1993.

10. Knowles MS. The Modern Practice of Adult Education: from Pedagogy to Andragogy. San Francisco : Jossey-Bass Publishers, 1980.

11. Kane MT. The Assessment of Medical Competence. Evaluation and the Health Professions. 1992; 15 : 163-182.

12. Dunn WR. Hamilton DD. The Critical Incident Technique - a brief guide. Medical Teacher. 1986; 8 : 207-215.

13. Delbec AL. Van de Ven AH. Gustafson DH. Group Techniques for Program Planning : a Guide to Nominal Group and Delphi Process. Glenview : Scott, Foresman and Company, 1975.

14. Gagné RM. Briggs LG. Wager WW. Principles of Instructional Design. New York: Holt, Rinehart and Wilson, 1988.

15. Stufflebeam DL. Foley WJ. Gephart WJ. Guba EG. Mammond RL. Merriman RL. Provus MM. Educational Evaluation and Decision Making. Itasca : FE. Peacock Publisher, 1971. 